\title{
RELIABILITY PROPERTIES OF RESIDUAL LIFE TIME AND INACTIVITY TIME OF SERIES AND PARALLEL SYSTEM
}

\author{
NITIN GUPTA, NEERAJ GANDOTRA AND RAKESH KUMAR BAJAJ
}

\begin{abstract}
The concepts of residual life time and inactivity time are extensively used in reliability theory for modeling life time data. In this paper we prove some new results on stochastic comparisons of residual life time and inactivity time in series and parallel systems. These results are in addition to the existing results of Li \& Zhang (2003) and Li \& Lu (2003). We also present sufficient conditions for aging properties of the residual life time and inactivity life time of series and parallel systems. Some examples from Weibull and Gompertz distributions are provided to support the results as well.
\end{abstract}

Mathematics Subject Classification 2000: 60E15, 62N05

Additional Key Words and Phrases: Likelihood ratio order, series system, parallel system.

\section{INTRODUCTION}

Let $X$ and $Y$ be two statistically independent random variables with an absolutely continuous distribution function $F(\cdot)$ and $G(\cdot)$, survival function $\bar{F}(\cdot)=1-F(\cdot)$ and $\bar{G}(\cdot)=1-G(\cdot)$ and probability density function $f(\cdot)$ and $g(\cdot)$ respectively. Suppose that

$$
\{x \in \mathbb{R}: f(x)>0\}=\{x \in \mathbb{R}: g(x)>0\}=(0, \infty)=S \text { (say) }
$$

where $\mathbb{R}=(-\infty, \infty)$. Let $X$ and $Y$ denote the lifetimes of two components, say $C_{1}$ and $C_{2}$. A series (parallel) system comprising of components $C_{1}$ and $C_{2}$ functions if and only if all (at least one) of its component function(s). Study of reliability properties of series and parallel systems is of importance to reliability engineers. Clearly $\min (X, Y)$ and $\max (X, Y)$ are respectively the lifetime of series and parallel systems comprising of components $C_{1}$ and $C_{2}$; here $\min (X, Y)(\max (X, Y))$ denotes the minimum (maximum) of $X$ and $Y$ respectively. The residual life of $X$ with age/time $t \geq 0$ is given by

$$
X_{t}=(X-t \mid X>t), t \geq 0
$$

and inactivity time of $X$ at time $t \geq 0$ is given by

$$
X_{(t)}=(t-X \mid X \leq t), t \geq 0
$$




\section{N. GUPTA, N. GANDOTRA AND R.K. BAJAJ}

For a fixed $t \geq 0$, the survival functions of $X_{t}$ and $X_{(t)}$ are given by

$$
S_{R, t}(x)=P\left(X_{t}>x\right)=\left\{\begin{array}{ll}
\frac{1}{\bar{F}(x+t)}, & \text { if } x<0 \\
\frac{\bar{F}(t)}{}, & \text { if } \geq 0
\end{array},\right.
$$

and

$$
S_{I, t}(x)=P\left(X_{(t)}>x\right)=\left\{\begin{array}{ll}
1 & \text { if } x<0 \\
\frac{F(t-x)}{F(t)}, & \text { if } 0 \leq x<t \\
0 & \text { if } x \geq t
\end{array},\right.
$$

respectively. Let $F_{R, t}(x)=1-S_{R, t}(x)$ and $F_{I, t}(x)=1-S_{I, t}(x)$ be the corresponding cumulative distribution functions. Block et. al. (1988), Chandra \& Roy (2001), Li \& Zhang (2003), Li \& Lu (2003), Li \& Zuo (2004), Misra et al. (2008) and Pellerey \& Petakos (2002) studied reliability properties of residual life/inactivity time. The stochastic comparisons of residual life time and inactivity time in series and parallel systems is discussed by Li and Lu (2003) and Li \& Zhang (2003). Note that

- the residual life of series (parallel) system having components $X$ and $Y$ is $(\min (X, Y))_{t}\left((\max (X, Y))_{t}\right)$;

- the inactivity time of series (parallel) system having components $X$ and $Y$ is $(\min (X, Y))_{(t)}\left((\max (X, Y))_{(t)}\right)$;

- the lifetime of the series (parallel) system having residual lives $X_{t}$ and $Y_{t}$ is $\min \left(X_{t}, Y_{t}\right)\left(\max \left(X_{t}, Y_{t}\right)\right)$;

- the lifetime of series (parallel) system having inactivity times $X_{(t)}$ and $Y_{(t)}$ is $\min \left(X_{(t)}, Y_{(t)}\right)\left(\left(\max \left(X_{(t)}, Y_{(t)}\right)\right)\right.$.

Let $\eta_{f}(x)=-f^{\prime}(x) / f(x), x \in S$, and $\eta_{g}(x)=-g^{\prime}(x) / g(x), x \in S$ denote the eta functions of random variable $X$ and $Y$ respectively. Glaser (1980) demonstrated that the eta functions play a vital role in the study of the failure rates. Throughout this paper, terms increasing and decreasing will be used for non-decreasing and non-increasing, respectively.

For the completeness in the presentation we include below some definitions of stochastic orders which are standard in the literature [See Shaked and Shanthiku$\operatorname{mar}(2007)]$.

Definition 1.1. The random variable $X$ is said to be smaller than random variable $Y$ in the

(a) likelihood ratio (lr) ordering $\left(X \leq_{l r} Y\right)$ if $\frac{g(x)}{f(x)}$ increases in $x \in S$;

(b) reversed failure rate (rfr) ordering $\left(X \leq_{r f r} Y\right)$ if $\frac{G(x)}{F(x)}$ increases in $x \in S$;

(c) usual stochastic (st) ordering $\left(X \leq_{s t} Y\right)$ if $\bar{F}(x) \leq \bar{G}(x)$, for all $x \in \mathbb{R}$.

Now we introduce some notions of aging (see Barlow and Proschan (1981)):

Definition 1.2. The random variable $X$ is said to have

(d) increasing failure rate (IFR) if the failure rate function $\frac{f(x)}{\bar{F}(x)}$ is increasing in $x \in S$; 
(e) decreasing failure rate (DFR) if the failure rate function $\frac{f(x)}{\bar{F}(x)}$ is decreasing in $x \in S$;

(f) decreasing reversed failure rate (DRFR) if the reversed failure rate function $\frac{f(x)}{F(x)}$ is decreasing in $x \in S$.

Now we provide a brief review of literature related to the results proved in the paper. Li \& Zhang (2003) proved that if $X$ and $Y$ are independent and identically distributed then, for all $t \geq 0,(\max (X, Y))_{t} \leq_{s t} \max \left(X_{t}, Y_{t}\right)$; similar results are also proved for inactivity time. Li \& Lu (2003) strengthen the results of Li \& Zhang (2003) and proved that if $X$ and $Y$ are independent and identically distributed then, for all $t \geq 0$,

(i) $(\max (X, Y))_{t} \leq_{l r} \max \left(X_{t}, Y_{t}\right)$;

(ii) $(\max (X, Y))_{(t)} \leq_{l r} \max \left(X_{(t)}, Y_{(t)}\right)$; and

(iii) $\min \left(X_{(t)}, Y_{(t)}\right) \leq_{l r}(\min (X, Y))_{(t)}$.

$\mathrm{Li} \& \mathrm{Lu}$ (2003) also proved that, if $X$ and $Y$ are independent (not necessarily identical distributed) then, for all $t \geq 0$,

(i) $(\max (X, Y))_{t} \leq_{f r} \max \left(X_{t}, Y_{t}\right)$;

(ii) $(\max (X, Y))_{(t)} \leq_{f r} \max \left(X_{(t)}, Y_{(t)}\right)$; and

(iii) $\min \left(X_{(t)}, Y_{(t)}\right) \leq_{f r}(\min (X, Y))_{(t)}$.

In section 2 of the paper, we obtain some new results on stochastic comparisons of residual life time and inactivity time in series and parallel systems. By assuming that $X$ and $Y$ are independent, but not necessarily identical distributed and letting $X \leq_{r f r} Y, \eta_{f}<0$ and $\eta_{g}>0$, (or $Y \leq_{r f r} X, \eta_{f}>0$ and $\eta_{g}>0$ ) we proved that the parallel system of used components, i.e., $\max \left(X_{t}, Y_{t}\right)$, is better than the used parallel system, i.e., $(\max (X, Y))_{t}$, in the sense of likelihood ratio order. Also by assuming $X$ and $Y$ are independent, but not necessarily identical distributed and letting $X \leq_{l r} Y$, (or $\left.Y \leq_{l r} X\right)$ we proved that, for any $t \geq 0$,

$$
(\max (X, Y))_{(t)} \leq_{f r} \max \left(X_{(t)}, Y_{(t)}\right) ;
$$

and

$$
\min \left(X_{(t)}, Y_{(t)}\right) \leq_{f r}(\min (X, Y))_{(t)} .
$$

In section 3, we proved various aging properties of used/inactive parallel/series systems and the parallel/series system of used/inactive components. Section 4 provides some examples relevant to the results provided in sections 2,3 .

\section{STOCHASTIC COMPARISON}

$\mathrm{Li} \& \mathrm{Lu}(2003)$ proved that if $X$ and $Y$ are independent and identical distributed then, for any $t \geq 0,(\max (X, Y))_{(t)} \leq_{l r} \max \left(X_{(t)}, Y_{(t)}\right)$. They also proved that if $X$ and $Y$ are independent, but not necessarily identically distributed then, for any $t \geq 0,(\max (X, Y))_{(t)} \leq_{f r} \max \left(X_{(t)}, Y_{(t)}\right)$. Here in the following theorem, we find the sufficient conditions for $(\max (X, Y))_{(t)} \leq_{l r} \max \left(X_{(t)}, Y_{(t)}\right)$ to hold when $X$ and $Y$ are independent, but not necessarily identically distributed. 
Theorem 2.1. If $X \leq_{l r} Y$ or $Y \leq_{l r} X$ then, for any $t \geq 0,(\max (X, Y))_{(t)} \leq_{l r}$ $\max \left(X_{(t)}, Y_{(t)}\right)$.

Proof. Let $t \geq 0$ be fixed. Let $H_{1, t}(x)$ and $h_{1, t}(x)$ denote respectively the cumulative distribution function and probability density function of random variable $(\max (X, Y))_{(t)}$. Then for $0 \leq x \leq t$,

$$
\begin{aligned}
H_{1, t}(x) & =P\left[(\max (X, Y))_{(t)} \leq x\right] \\
& =\frac{F(t) G(t)-F(t-x) G(t-x)}{F(t) G(t)},
\end{aligned}
$$

and

$$
h_{1, t}(x)=\frac{F(t-x) g(t-x)+f(t-x) G(t-x)}{F(t) G(t)} .
$$

Let $M_{1, t}(x)$ and $m_{1, t}(x)$ denote the cumulative distribution function and probability density function of random variable $\max \left(X_{(t)}, Y_{(t)}\right)$. We have, for $0 \leq x \leq t$,

$$
\begin{aligned}
M_{1, t}(x) & =P\left(\left(\max \left(X_{(t)}, Y_{(t)}\right)\right) \leq x\right) \\
& =\left(\frac{F(t)-F(t-x)}{F(t)}\right)\left(\frac{G(t)-G(t-x)}{G(t)}\right),
\end{aligned}
$$

and

$$
m_{1, t}(x)=\frac{(F(t)-F(t-x)) g(t-x)+(G(t)-G(t-x)) f(t-x)}{F(t) G(t)} .
$$

Consider, for $0 \leq x<t$,

$$
\begin{aligned}
R_{1, t}(x) & =\frac{m_{1, t}(x)}{h_{1, t}(x)} \\
& =\frac{(F(t)-F(t-x)) g(t-x)+(G(t)-G(t-x)) f(t-x)}{F(t-x) g(t-x)+f(t-x) G(t-x)} \\
& =-1+\frac{F(t) g(t-x)+G(t) f(t-x)}{F(t-x) g(t-x)+f(t-x) G(t-x)}
\end{aligned}
$$

It is easy to verify that, for $0 \leq x<t$,

$$
\begin{aligned}
R_{1, t}^{\prime}(x)= & \frac{f(t-x) g(t-x)}{[F(t-x) g(t-x)+f(t-x) G(t-x)]^{2}}[2 F(t) g(t-x)+2 G(t) f(t-x) \\
& \left.+\left[\left\{\eta_{f}(t-x)-\eta_{g}(t-x)\right\}\{G(t) F(t-x)-G(t-x) F(t)\}\right]\right] .
\end{aligned}
$$

We will prove the assertion for the case $X \leq_{l r} Y$ as for the case $Y \leq_{l r} X$ the assertion follows similarly. Note that,

$$
X \leq_{l r} Y \Leftrightarrow \ln \left(\frac{g(t)}{f(t)}\right) \text { is increasing in } t \in(0, \infty) \Leftrightarrow \eta_{f}(t) \geq \eta_{g}(t), \forall t>0 .
$$

Also,

$$
X \leq_{l r} Y \Rightarrow X \leq_{r f r} Y \Leftrightarrow F(u) G(v) \geq F(v) G(u), \forall 0 \leq u \leq v<\infty .
$$

Using (2.6) and (2.7) in (2.5), we conclude that $R_{1, t}^{\prime}(x) \geq 0, \forall 0 \leq x<t$, i.e., $(\max (X, Y))_{(t)} \leq_{l r} \max \left(X_{(t)}, Y_{(t)}\right)$. 
The following corollary is an immediate consequence of Theorem 2.1.

Corollary 1. If $X={ }_{s t} Y$, then $(\max (X, Y))_{(t)} \leq_{l r} \max \left(X_{(t)}, Y_{(t)}\right)$.

Remark 1. The result stated in Corollary 1 is in by Li and Lu (2003).

Li \& Lu (2003) proved that if $X$ and $Y$ are independent and identical distributed then, for any $t \geq 0,(\min (X, Y))_{(t)} \geq_{l r} \min \left(X_{(t)}, Y_{(t)}\right)$. They also proved that if $X$ and $Y$ are independent, but not necessarily identically distributed then, for any $t \geq 0,(\min (X, Y))_{(t)} \geq_{f r} \min \left(X_{(t)}, Y_{(t)}\right)$. Here in the following theorem, we find the sufficient conditions for $(\min (X, Y))_{(t)} \geq_{l r} \min \left(X_{(t)}, Y_{(t)}\right)$ to hold when $X$ and $Y$ are independent, but not necessarily identically distributed.

Theorem 2.2. If $X \leq_{l r} Y$ or $Y \leq_{l r} X$ then, for any $t \geq 0,(\min (X, Y))_{(t)} \geq_{l r}$ $\min \left(X_{(t)}, Y_{(t)}\right)$.

Proof. Fix $t \geq 0$. Let $H_{2, t}(x)$ and $h_{2, t}(x)$ denote respectively the cumulative distribution function and probability density function of random variable $(\min (X, Y))_{(t)}$. Then, for $0 \leq x \leq t$,

$$
\begin{aligned}
H_{2, t}(x) & =P\left((\min (X, Y))_{(t)} \leq x\right) \\
& =\frac{(1-F(t-x))(1-G(t-x))-(1-F(t))(1-G(t))}{1-(1-F(t))(1-G(t))},
\end{aligned}
$$

and

$$
h_{2, t}(x)=\frac{(1-F(t-x)) g(t-x)+(1-G(t-x)) f(t-x)}{1-(1-F(t))(1-G(t))} .
$$

For $0 \leq x \leq t$, let $M_{2, t}(x)$ and $m_{2, t}(x)$ denote respectively the cumulative distribution function and probability density function of random variable $\min \left(X_{(t)}, Y_{(t)}\right)$. Then, for $0 \leq x \leq t$,

$$
\begin{aligned}
M_{2, t}(x) & =P\left(\min \left(X_{(t)}, Y_{(t)}\right) \leq x\right) \\
& =1-\frac{F(t-x) G(t-x)}{F(t) G(t)},
\end{aligned}
$$

and

$$
m_{2, t}(x)=\frac{f(t-x) G(t-x)+F(t-x) g(t-x)}{F(t) G(t)} .
$$

Consider, for $0 \leq x<t$,

$$
\begin{aligned}
R_{2, t}(x) & =\frac{h_{2, t}(x)}{m_{2, t}(x)} \\
& =\left(\frac{F(t) G(t)}{1-(1-F(t))(1-G(t))}\right)\left(\frac{(1-F(t-x)) g(t-x)+(1-G(t-x)) f(t-x)}{F(t-x) g(t-x)+f(t-x) G(t-x)}\right) \\
& =A(t) Z_{t}(x),
\end{aligned}
$$

where

$$
A(t)=\frac{F(t) G(t)}{1-(1-F(t))(1-G(t))}
$$




\section{N. GUPTA, N. GANDOTRA AND R.K. BAJAJ}

and, for $0 \leq x<t$,

$$
Z_{t}(x)=-1+\frac{g(t-x)+f(t-x)}{F(t-x) g(t-x)+f(t-x) G(t-x)} .
$$

Clearly, for $0 \leq x<t$,

$$
\begin{aligned}
& Z_{t}^{\prime}(x)= \frac{1}{[F(t-x) g(t-x)+G(t-x) F(t-x)]^{2}}[-[F(t-x) g(t-x)+f(t-x) G(t-x)] \\
& {\left[g^{\prime}(t-x)+f^{\prime}(t-x)\right]+[g(t-x)+f(t-x)]\left[F(t-x) g^{\prime}(t-x)+f(t-x) g(t-x)\right.} \\
&\left.\left.\quad+f^{\prime}(t-x) G(t-x)+g(t-x) f(t-x)\right]\right] \\
&= \frac{1}{[F(t-x) g(t-x)+G(t-x) f(t-x)]^{2}}\left[2 g^{2}(t-x) f(t-x)+2 f^{2}(t-x) g(t-x)\right. \\
& \quad+\left[F(t-x)\left\{-g(t-x) f^{\prime}(t-x)+f(t-x) g^{\prime}(t-x)\right\}\right]+\left[G ( t - x ) \left\{g(t-x) f^{\prime}(t-x)\right.\right. \\
&\left.\left.\left.\quad-f(t-x) g^{\prime}(t-x)\right\}\right]\right] \\
&= \frac{f(t-x) g(t-x)}{[F(t-x) g(t-x)+G(t-x) f(t-x)]^{2}}[2 g(t-x)+2 f(t-x) \\
&\left.\quad+\left[\left\{\eta_{f}(t-x)-\eta_{g}(t-x)\right\}\{F(t-x)-G(t-x)\}\right]\right] .
\end{aligned}
$$

We will prove the assertion for the case $X \leq_{l r} Y$ as for the case $Y \leq_{l r} X$ the assertion follows similarly. Note that, as in proof of Theorem 2.1,

$$
X \leq_{l r} Y \Leftrightarrow \eta_{f}(t) \geq \eta_{g}(t), \forall t>0 .
$$

Also,

$$
X \leq_{l r} Y \Rightarrow X \leq_{s t} Y \Leftrightarrow F(u) \geq G(u), \forall 0 \leq u<\infty .
$$

Using (2.13) and (2.14) in (2.12), we conclude that $Z_{t}^{\prime}(x) \geq 0, \forall 0 \leq x<t$, i.e., $(\min (X, Y))_{(t)} \geq_{l r} \min \left(X_{(t)}, Y_{(t)}\right)$.

The following corollary is an immediate consequence of Theorem 2.2.

Corollary 2. If $X={ }_{s t} Y$, then $(\min (X, Y))_{(t)} \geq_{l r} \min \left(X_{(t)}, Y_{(t)}\right)$.

Remark 2. The result stated in Corollary 2 is in by Li and Lu (2003).

Li \& Lu (2003) proved that if $X$ and $Y$ are independent and identical distributed then, for any $t \geq 0,(\max (X, Y))_{t} \leq_{l r} \max \left(X_{t}, Y_{t}\right)$. They also proved that if $X$ and $Y$ are independent, but not necessarily identically distributed then, for any $t \geq 0,(\max (X, Y))_{t} \leq_{f r} \max \left(X_{t}, Y_{t}\right)$. Here in the following theorem, we find the sufficient conditions for $(\max (X, Y))_{t} \leq_{l r} \max \left(X_{t}, Y_{t}\right)$ to hold when $X$ and $Y$ are independent, but not necessarily identically distributed.

TheOREm 2.3. If $X \leq_{r f r} Y, \eta_{f} \leq 0$ and $\eta_{g} \geq 0$ or $Y \leq_{r f r} X, \eta_{f} \geq 0$ and $\eta_{g} \leq 0$ then, for any $t \geq 0,(\max (X, Y))_{t} \leq_{l r} \max \left(X_{t}, Y_{t}\right)$.

Proof. Let $t \geq 0$ be fixed. Let $H_{3, t}(x)$ and $h_{3, t}(x)$ denote respectively the cumulative distribution function and probability density function of random variable 
$(\max (X, Y))_{t}$. Then for $x \geq 0$,

$$
\begin{aligned}
H_{3, t}(x) & =P\left[(\max (X, Y))_{t} \leq x\right] \\
& =\frac{F(t+x) G(t+x)-F(t) G(t)}{1-F(t) G(t)},
\end{aligned}
$$

and

$$
h_{3, t}(x)=\frac{f(t+x) G(t+x)+g(t+x) F(t+x)}{1-F(t) G(t)} .
$$

Let $M_{3, t}(x)$ and $m_{3, t}(x)$ denote the cumulative distribution function and probability density function of random variable $\max \left(X_{t}, Y_{t}\right)$. We have, for $x \geq 0$,

$$
\begin{aligned}
M_{3, t}(x) & =P\left(\left(\max \left(X_{t}, Y_{t}\right)\right) \leq x\right) \\
& =\left(\frac{F(t+x)-F(t)}{1-F(t)}\right)\left(\frac{G(t+x)-G(t)}{1-G(t)}\right),
\end{aligned}
$$

and

$$
m_{3, t}(x)=\frac{(G(t+x)-G(t)) f(t+x)+(F(t+x)-F(t)) g(t+x)}{(1-F(t))(1-G(t))} .
$$

Consider, for $x \geq 0$,

$$
\begin{aligned}
R_{3, t}(x) & =\frac{m_{3, t}(x)}{h_{3, t}(x)} \\
& =\left(\frac{1-F(t) G(t)}{(1-F(t))(1-G(t))}\right)\left(\frac{(G(t+x)-G(t) f(t+x))+(F(t+x)-F(t) g(t+x))}{(1-F(t))(1-G(t))}\right) \\
& =B(t) U_{t}(x),
\end{aligned}
$$

where

$$
B(t)=\frac{1-F(t) G(t)}{(1-F(t))(1-G(t))},
$$

and

$$
U_{t}(x)=1-\frac{f(t+x) G(t)+g(t+x) F(t)}{f(t+x) G(t+x)+g(t+x) F(t+x)} .
$$

It is easy to verify that, for $x \geq 0$,

$$
\begin{aligned}
& U_{t}^{\prime}(x)=\frac{f(t+x) g(t+x)}{[f(t+x) G(t+x)+g(t+x) F(t+x)]^{2}}[2(G(t) f(t+x)+g(t+x) F(t)) \\
& \left.+(G(t+x) F(t)-G(t) F(t+x))\left(\eta_{g}(t+x)-\eta_{f}(t+x)\right)\right]
\end{aligned}
$$

We will prove the assertion for the case $X \leq_{r f r} Y, \eta_{f} \leq 0$ and $\eta_{g} \geq 0$ as for the case $Y \leq_{r f r} X, \eta_{f} \geq 0$ and $\eta_{g} \leq 0$ the assertion follows similarly. Note that,

$$
X \leq_{r f r} Y \Leftrightarrow F(u) G(v) \geq F(v) G(u), \forall 0 \leq u \leq v<\infty .
$$

Now using (2.20), $\eta_{f} \leq 0$ and $\eta_{g} \geq 0$ in (2.19), we conclude that $U_{t}^{\prime}(x) \geq 0, \forall x \geq 0$, i.e., $(\max (X, Y))_{t} \geq_{l r} \max \left(X_{t}, Y_{t}\right)$. 


\section{AGEING PROPERTIES:}

In this section we discuss the aging properties of the residual life time and inactivity time in series and parallel systems. The following property proves that if the random variables $X$ and $Y$ have DRFR, then this property is preserved by the random variable $\max \left(X_{t}, Y_{t}\right)$.

Property 1. Suppose that the random variables $X$ and $Y$ have DRFR. Then, for any $t \geq 0$, the random variable $\max \left(X_{t}, Y_{t}\right)$ has DRFR.

Proof. Fix $t>0$. Let $\lambda_{t}(x)$ and $\mu_{t}(x)$ denote respectively the reversed failure rates of $X_{t}$ and $Y_{t}$ and let $M_{4, t}(x)$ denote the cumulative distribution function of $\max \left(X_{t}, Y_{t}\right)$. Let $F_{R, t}(x)$ and $G_{R, t}(x)$ denote respectively the cumulative distribution functions of $X_{t}$ and $Y_{t}$. Then, for $x \geq 0$,

$\lambda_{t}(x)=\frac{f(x+t)}{F(x+t)-F(t)}, \mu_{t}(x)=\frac{g(x+t)}{G(x+t)-G(t)}$, and $M_{4, t}(x)=F_{R, t}(x) G_{R, t}(x)$.

$X$ has DRFR implies that $F(x) f^{\prime}(x) \leq f^{2}(x), \forall x>0$, which in turn implies that $\lambda_{t}^{\prime}(x) \leq 0, \forall x>0$ (i.e., $X_{t}$ has DRFR or equivalently $\ln \left(F_{R, t}(x)\right)$ is concave in $x \in(0, \infty))$. Similarly $Y$ has DRFR implies that $Y_{t}$ has DRFR (i.e., $\ln \left(G_{R, t}(x)\right)$ is concave in $x \in(0, \infty))$. Thus if $X$ and $Y$ have DRFR then

$$
\ln \left(M_{4, t}(x)\right)=\ln \left(F_{R, t}(x) G_{R, t}(x)\right)=\ln \left(F_{R, t}(x)\right)+\ln \left(G_{R, t}(x)\right)
$$

is concave in $x \in(0, \infty)$, i.e., $\max \left(X_{t}, Y_{t}\right)$ has DRFR.

In the following property we prove that if the random variables $X$ and $Y$ have DRFR, then the random variable $(\max (X, Y))_{(t)}$ has IFR.

Property 2. Suppose that the random variables $X$ and $Y$ have DRFR. Then, for any $t \geq 0$, the random variable $(\max (X, Y))_{(t)}$ has $\operatorname{IFR}$.

Proof. Fix $t \geq 0$. Obviously if random variables $X$ and $Y$ have DRFR, then $\max (X, Y)$ also has DRFR. Also it is easy to verify that if a non-negative random variable $Z$ has DRFR then, for any $s \geq 0$, the random variable $Z_{(s)}=(s-Z \mid Z \leq s)$ has IFR. Thus under the hypothesis of the theorem, $\max (X, Y)$ has DRFR, which in turn implies that $(\max (X, Y))_{(t)}$ has IFR.

In the following property we prove that if the random variables $X$ and $Y$ have IFR, then the random variable $\max \left(X_{(t)}, Y_{(t)}\right)$ has DRFR.

Property 3. Suppose that the random variables $X$ and $Y$ have IFR. Then, for any $t \geq 0$, the random variable $\max \left(X_{(t)}, Y_{(t)}\right)$ has DRFR.

Proof. Fix $t \geq 0$. Obviously if random variables $X$ and $Y$ have IFR, then random variables $X_{(t)}$ and $Y_{(t)}$ have DRFR. This in turn implies that $\max \left(X_{(t)}, Y_{(t)}\right)$ has DRFR.

In the following property we prove that if the random variables $X$ and $Y$ have DRFR, then the random variable $\min \left(X_{(t)}, Y_{(t)}\right)$ has IFR.

Property 4. Suppose that the random variables $X$ and $Y$ have DRFR. Then, for any $t \geq 0$, the random variable $\min \left(X_{(t)}, Y_{(t)}\right)$ has IFR. 
Proof. Fix $t \geq 0$. Obviously if random variables $X$ and $Y$ have DRFR, then random variables $X_{(t)}$ and $Y_{(t)}$ have IFR. This in turn implies that $(\min (X, Y))_{(t)}$ has IFR.

The following property proves that if the random variables $X$ and $Y$ have IFR, then this property is preserved by the random variable $\min \left(X_{t}, Y_{t}\right)$.

Property 5. Suppose that the random variables $X$ and $Y$ have IFR. Then, for any $t \geq 0$, the random variable $\min \left(X_{t}, Y_{t}\right)$ has IFR.

Proof. Fix $t \geq 0$. Obviously if random variables $X$ and $Y$ have IFR, then random variables $X_{t}$ and $Y_{t}$ have IFR. This in turn implies that $\min \left(X_{t}, Y_{t}\right)$ has IFR.

The following property proves that if the random variables $X$ and $Y$ have IFR, then this property is preserved by the random variable $(\min (X, Y))_{t}$.

Property 6. Suppose that the random variables $X$ and $Y$ have IFR. Then, for any $t \geq 0$, the random variable $(\min (X, Y))_{t}$ has $I F R$.

Proof. Fix $t \geq 0$. Obviously if random variables $X$ and $Y$ have IFR, then $\min (X, Y)$ also has IFR. Also it is easy to verify that if a non-negative random variable $Z$ has IFR then, for any $s \geq 0$, the random variable $Z_{s}=(Z-s \mid Z>s)$ has IFR. Thus under the hypothesis of the theorem, $\min (X, Y)$ has IFR, which in turn implies that $(\min (X, Y))_{t}$ has IFR.

\section{EXAMPLES}

Weibull and Gompertz distribution are important life distributions which are used in reliability modeling. In this section we provide some examples relevant to the theory developed in Sections 2, 3. For a survey of these distributions one may refer Marshall and Olkin (2007).

\section{Weibull distribution}

Consider that the random variable $X$ has Weibull distribution with parameters $(\alpha, \lambda)$ and with survival function

$$
\bar{F}(x)=e^{-(\lambda x)^{\alpha}}, x>0, \lambda>0, \alpha>0 .
$$

Then, its probability density function is

$$
f(x)=\alpha \lambda^{\alpha} x^{\alpha-1} e^{-(\lambda x)^{\alpha}}, x>0, \lambda>0, \alpha>0,
$$

and

$$
f^{\prime}(x)=\alpha \lambda^{\alpha} x^{\alpha-2} e^{-(\lambda x)^{\alpha}}\left((\alpha-1)-\alpha x^{\alpha} \lambda^{\alpha}\right) .
$$

Clearly $f^{\prime}(x) \leq 0$, if $\alpha \leq 1$. Similarly let $Y$ follows Weibull $(\beta, \mu)$. Note that $f^{\prime} \leq 0$ and $g^{\prime} \leq 0 \Leftrightarrow F$ and $G$ are concave $\Rightarrow \ln F$ and $\ln G$ are concave $\Leftrightarrow X$ and $Y$ have DRFR. Hence if $X$ and $Y$ follows $\operatorname{Weibull}(\alpha, \lambda), \alpha \leq 1$, and $\operatorname{Weibull}(\beta, \mu), \beta \leq 1$, then sufficient conditions of Property 1, 2 and 4 are satisfied.

It is well known that the random variable $X$, which follows $\operatorname{Weibull}(\alpha, \lambda)$, has IFR if $\alpha \geq 1$ (see Barlow and Proschan (1981)). Therefore, if $X$ and $Y$ follows 


\section{N. GUPTA, N. GANDOTRA AND R.K. BAJAJ}

Weibull $(\alpha, \lambda) \alpha \geq 1$ and Weibull $(\beta, \mu), \beta \geq 1$, then sufficient conditions of Property 3,5 and 6 are satisfied.

To observe when $X \leq_{l r} Y\left(Y \leq_{l r} X\right)$, consider

$$
\frac{g(x)}{f(x)}=\frac{\beta}{\alpha} \frac{\mu^{\beta}}{\lambda^{\alpha}} \psi(x),
$$

where $\psi(x)=x^{\beta-\alpha} e^{(\lambda x)^{\alpha}} e^{-(\mu x)^{\beta}}$. Further

$$
\begin{aligned}
\psi^{\prime}(x) & =x^{\beta-\alpha-1} e^{(\lambda x)^{\alpha}} e^{-(\mu x)^{\beta}}\left((\beta-\alpha)+\alpha \lambda^{\alpha} x^{\alpha}-\beta \mu^{\beta} x^{\beta}\right) \\
& =x^{\beta-\alpha-1} e^{(\lambda x)^{\alpha}} e^{-(\mu x)^{\beta}}\left(\alpha\left(\lambda^{\alpha} x^{\alpha}-1\right)+\beta\left(1-\mu^{\beta} x^{\beta}\right)\right),
\end{aligned}
$$

if we take $\alpha=\beta, \lambda \geq \mu(\alpha=\beta, \lambda \leq \mu)$, then $\psi^{\prime}(x) \geq 0\left(\psi^{\prime}(x) \leq 0\right)$, i.e., $X \leq_{l r} Y$ $\left(Y \leq{ }_{l r} X\right)$.

Hence if $X$ and $Y$ follows Weibull $(\alpha, \lambda)$ and Weibull $(\beta, \mu)$, respectively such that $\alpha=\beta$, it is clear from above arguments (also see Theorems 2.1 and 2.2) that,

$$
(\max (X, Y))_{(t)} \leq_{l r} \max \left(X_{(t)}, Y_{(t)}\right)
$$

and

$$
(\min (X, Y))_{(t)} \geq_{l r} \min \left(X_{(t)}, Y_{(t)}\right)
$$

Gompertz distribution

If we consider the random variable $X$ has Gompertz distribution with scale parameter $\lambda$ and frailiy parameter $\xi$, i.e., $X$ follows $\operatorname{Gompertz}(\lambda, \xi)$. Then, the random variable $X$ has survival function

$$
\bar{F}(x)=e^{-\xi\left(e^{\lambda x}-1\right)}, x \geq 0, \lambda \geq 0, \xi \geq 0,
$$

its probability density function is

$$
f(x)=\lambda \xi e^{\lambda x-\xi\left(e^{\lambda x}-1\right)}, x \geq 0, \lambda \geq 0, \xi \geq 0,
$$

and

$$
f^{\prime}(x)=\lambda^{2} \xi e^{\lambda x-\xi\left(e^{\lambda x}-1\right)}\left(1-\xi e^{\lambda x}\right) .
$$

On applying the Maclaurin's series to $e^{\lambda x}$ in expression (4.1), we have

$$
f^{\prime}(x)=\lambda^{2} \xi e^{\lambda x-\xi\left(e^{\lambda x}-1\right)}\left(1-\xi\left(1+\lambda x+\frac{(\lambda x)^{2}}{2 !}+\ldots\right)\right)
$$

If we choose $\xi>1$, then $f^{\prime}(x) \leq 0$. Similarly let $Y$ follows $\operatorname{Gompertz}(\mu, \eta)$. Note that $f^{\prime} \leq 0$ and $g^{\prime} \leq 0 \Leftrightarrow F$ and $G$ are concave $\Rightarrow \ln F$ and $\ln G$ are concave $\Leftrightarrow X$ and $Y$ have DRFR. Clearly if $X$ and $Y$ follows $\operatorname{Gompertz}(\lambda, \xi), \xi>1$, and $\operatorname{Gompertz}(\mu, \eta), \eta>1$, respectively, then sufficient conditions of Property 1, 2 and 4 are satisfied.

To observe when $X \leq_{l r} Y\left(Y \leq_{l r} X\right)$ consider for the case when $\lambda=\mu$,

$$
\begin{aligned}
\frac{g(x)}{f(x)} & =\frac{\eta e^{-\eta\left(e^{\lambda x}-1\right)}}{\xi e^{-\xi\left(e^{\lambda x}-1\right)}} \\
& =\frac{\eta}{\xi} e^{\left(e^{\lambda x}-1\right)(\xi-\eta)}
\end{aligned}
$$


which is increasing (decreasing) in $x$ if $\xi \geq \eta(\xi \leq \eta)$. Therefore if we take $\lambda=\mu$, $\xi \geq \eta(\lambda=\mu, \xi \leq \eta)$ then $X \leq_{l r} Y\left(Y \leq_{l r} X\right)$.

Hence if $X$ and $Y$ follows $\operatorname{Gompertz}(\lambda, \xi)$ and $\operatorname{Gompertz}(\mu, \eta)$ respectively such that $\lambda=\mu$, it is clear from above arguments (also see Theorems 2.1 and 2.2) that,

$$
(\max (X, Y))_{(t)} \leq_{l r} \max \left(X_{(t)}, Y_{(t)}\right)
$$

and

$$
(\min (X, Y))_{(t)} \geq_{l r} \min \left(X_{(t)}, Y_{(t)}\right)
$$

\section{CONCLUSION}

The stochastic comparison of residual life and inactivity time of series and parallel systems had been studied in the literature when the random variables are independent and identically distributed. In this paper such results are extended when the condition of identical distribution is omitted. By assuming that $X$ and $Y$ are independent, but not necessarily identical distributed and letting $X \leq_{l r} Y, \eta_{f} \leq 0$ and $\eta_{g} \geq 0,\left(\right.$ or $Y \leq_{l r} X, \eta_{f} \geq 0$ and $\eta_{g} \leq 0$ ) we proved that the parallel system of used components, i.e., $\max \left(X_{t}, Y_{t}\right)$, is better than the used parallel system, i.e., $(\max (X, Y))_{t}$, in the sense of likelihood ratio order. Also by assuming $X$ and $Y$ are independent, but not necessarily identical distributed and letting $X \leq_{l r} Y$, (or $\left.Y \leq_{l r} X\right)$ we proved that, for any $t \geq 0$,

$$
(\max (X, Y))_{(t)} \leq_{f r} \max \left(X_{(t)}, Y_{(t)}\right)
$$

and

$$
\min \left(X_{(t)}, Y_{(t)}\right) \leq_{f r}(\min (X, Y))_{(t)} .
$$

Also, we proved various aging properties of used/inactive parallel/series systems and the parallel/series system of used/inactive components. These results are supported by well known distributions, such as Weibull and Gompertz distributions.

\section{Acknowledgment}

The authors would like to sincerely thank two anonymous reviewers for their constructive comments to improve the work presented in the paper. The authors also acknowledge Professor Vladimír Ďurikovič, Editor in Chief, Journal of Applied Mathematics, Statistics and Informatics (JAMSI).

\section{REFERENCES}

[1] Barlow, R.E.; Proschan, F. Statistical theory of reliability and life testing. Silver Spring, MD: Madison, 1981.

[2] Block, H.; Savits, T.; Singh, H. The reversed hazard rate function. Probability in the Engineering and informational Sciences, 1998, 12, 69-70.

[3] Chandra, N.K.; Roy, D. Some results on reversed hazard rate. Probability in the Engineering and informational Sciences, 2001, 15, 95-102.

[4] Glaser R.E. Bathtub and related failure rate characterizations. Journal of American Statistical Association, 1980, 75, 667-672.

[5] Li, X.; Lu, J. Stochastic comparison on residual life and inactivity time of series and parallel systems. Probability in the Engineering and informational Sciences, 2003, 17, 267-275.

[6] Li, X.; Zhang, S. Comparision between a system of used components and a used system. Journal of Lanzhou University, 2003. 


\section{N. GUPTA, N. GANDOTRA AND R.K. BAJAJ}

[7] Li, X.; Zuo, M.J. Stochastic comparisons of residual life and inactivity time at a random time. Stochastic Models, 2004, 20 (2), 229-235.

[8] Misra N.; Gupta N.; Dhariyal I.D. Stochastic Properties of Residual Life and Inactivity Time at a Random Time. Stochastic Models, 2008, 24 (1), 89-102.

[9] Marshall A., Olkin I. Life Distributions, Part IV, Springer, 2007.

[10] Pellerey F.; Petakos, K. On the closure property of the NBUC class under the formation of parallel systems. IEEE Transactions on Relaibility, 2002, 51(4),452-454.

[11] Shaked, M., Shanthikumar, J. G. Stochastic Orders, Springer, 2007.

Nitin Gupta,

Department of Mathematics,

Jaypee University of Information Technology,

Waknaghat, Solan (H.P.) Pin - 173234 INDIA.

email: nitin.gupta@juit.ac.in

Neeraj Gandotra,

Department of Mathematics,

Jaypee University of Information Technology,

Waknaghat, Solan (H.P.) Pin - 173234 INDIA.

email: neeraj.juit@gmail.com

Rakesh Kumar Bajaj,

Department of Mathematics,

Jaypee University of Information Technology,

Waknaghat, Solan (H.P.) Pin - 173234 INDIA.

email: rakesh.bajaj@gmail.com

Received April 2011 